\title{
A New Class of Mixed-Valence Systems with Orbitally Degenerate Organic Redox Centers. Examples Based on Hexa-Rhenium Molecular Prisms
}

\author{
Peter H. Dinolfo, ${ }^{\dagger \neq}$ Veaceslav Coropceanu,,$^{\S}$ Jean-Luc Brédas, ${ }^{\S}$ and Joseph T. Hupp ${ }^{*}, \dagger$ \\ Department of Chemistry, Northwestern University, Evanston, Illinois 60208, and School of Chemistry and \\ Biochemistry, Georgia Institute of Technology, Atlanta, Georgia 30332 \\ Received April 6, 2006; E-mail: jthupp@chem.northwestern.edu
}

The experimental and theoretical investigation of mixed-valence (MV) compounds has yielded a tremendous amount of information regarding intramolecular electron transfer reactions. The most common examples of MV systems are those of the type $\mathrm{M}^{n}$-bridge$\mathrm{M}^{n+1}$ where $\mathrm{M}$ is a transition metal and the bridge is an organic linker, ${ }^{1}$ although many examples of purely organic systems exist. ${ }^{2}$

Here, we report the MV properties of two supramolecular complexes: triangular prisms of the form $\left(\left[\operatorname{Re}(\mathrm{CO})_{3}\right]_{2} \mathrm{X}\right)_{3}-\mu, \mu^{\prime}, \mu^{\prime \prime}-$ [tPyTz $]_{2}$ where $\mathrm{X}$ is $2,2^{\prime}$-bisbenzimidazolate (1) or a pair of benzylthiols (2), and tPyTz is tri-(4-pyridyl)-1,3,5-triazine. As in the case of recently reported rectangular analogues, that is, $\left(\left[\operatorname{Re}(\mathrm{CO})_{3}\right]_{2} \mathrm{X}\right)_{2^{-}}$ $\mu, \mu^{\prime}$-[LL $]_{2}$ assemblies (LL is a redox-active linear dipyridyl ligand), ${ }^{3}$ the reduction of $\mathbf{1}$ or $\mathbf{2}$ by one electron produces a ligand-centered mixed-valence (LCMV) state where the azine ligands are the redox centers. As with the rectangular assemblies, the Re atoms play solely a structural role and do not mediate electronic communication between IPyTz units. Rather, communication is achieved by direct overlap of donor and acceptor orbitals. ${ }^{3}$

Surprisingly, the prisms display significant differences in their MV properties as compared to the rectangles. These are revealed in electroabsorbance (Stark) responses (see below) and are attributed to effects that originate in orbital degeneracy of the $\mathbf{t P y T z}$ redox sites. Indeed, the $D_{3 h}$ symmetry of the tPyTz unit leads to double degeneracy of its LUMO level; the addition of one electron to such a level leads to a 2 -fold degenerate reduced state. Thus, $\mathbf{1}^{-}$and $\mathbf{2}^{-}$ represent rare (to our knowledge the first organic) examples of MV systems with redox centers possessing orbitally degenerate electronic states.

1 and $\mathbf{2}$ were synthesized via methods similar to those of the rectangles; see Supporting Information. An ORTEP drawing for the X-ray single-crystal structure of $\mathbf{1}$ is shown in Figure 1 . The addition of a third di-Re column unit to the structure is sufficient to align the tPyTz ligands to within $1 \AA$ of each other laterally. The $\mathrm{Re}-\mathrm{Re}$ distances on the short sides of the prisms average 5.75 $\AA$, while the tPyTz ligands bow inward to give a minimum separation distance of $3.3 \AA$ for the redox-active triazine ringsdistortions that are similar to those of tetra-rhenium rectangles. ${ }^{3 a, c}$

Cyclic voltammograms of the prisms feature multiple oneelectron waves corresponding to reversible stepwise addition of electrons to cofacial tPyTz units. $\mathbf{1}$ is reduced at $-0.97,-1.25$, and $-1.73 \mathrm{~V}$ versus ferrocene; 2 reduces at $-1.15,-1.40,-1.82$, and $-2.23 \mathrm{~V}$. The potential differences are measures of MV stability and yield comproportionation constants of $5 \times 10^{4}, 1.7 \times 10^{4}$, and $7 \times 10^{6}$ for $\mathbf{1}^{-}, \mathbf{2}^{-}$, and $\mathbf{2}^{3-}$, respectively.

Assignment of the reductions above as tPyTz centered was confirmed by vis-NIR spectroelectrochemical measurements. Absorption spectra for the singly and doubly reduced forms of $\mathbf{1}$ and $\mathbf{2}$ are nearly identical. Reduction to the $1-$ state of either is

$\dagger$ Northwestern University.

¥ Present address: Department of Chemistry, Stanford University, Stanford, CA

94306 .

\$ Georgia Institute of Technology.

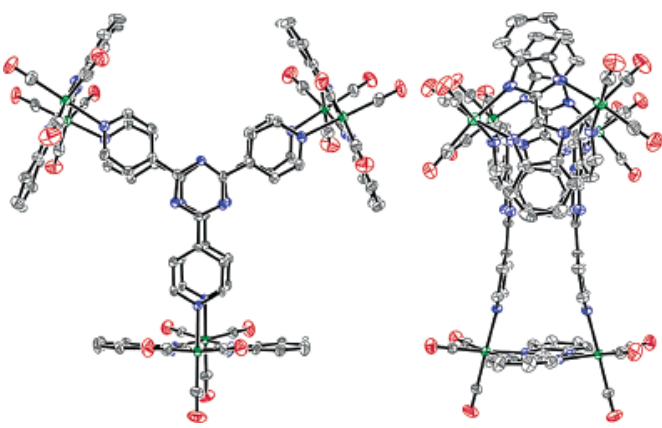

Figure 1. ORTEP drawings for the crystal structure of $\mathbf{1}$ (top and side views). C, black; $\mathrm{N}$, blue; $\mathrm{O}$, red; and $\mathrm{Re}$, green. $\mathrm{H}$ atoms have been removed for clarity. Displacement ellipsoids are drawn at $50 \%$ probability.

accompanied by the appearance of absorption bands at 600,850 , and $960 \mathrm{~nm}$. These increase in intensity upon reduction to the 2state and are assigned as tPyTz anion-centered transitions.

In addition, the singly reduced forms of $\mathbf{1}$ and $\mathbf{2}$ (MV species) show broad, Gaussian bands centered at 5650 and $5500 \mathrm{~cm}^{-1}$, respectively. These bands are not present in either the neutral or doubly reduced state and are assigned as tPyTz-based intervalence transitions (IT). The bands are fairly intense $(\epsilon=3400$ and 4000 $\mathrm{M}^{-1} \mathrm{~cm}^{-1}$ for $\mathbf{1}^{-}$and $\mathbf{2}^{-}$, respectively) and somewhat narrower than predicted by the Hush model for fully valence localized species (2550 and $2565 \mathrm{~cm}^{-1}$ versus 3600 and $3560 \mathrm{~cm}^{-1}$ for $\mathbf{1}^{-}$and $\mathbf{2}^{-}$, respectively). ${ }^{4}$ Both of these properties are hallmarks of strongly coupled MV species. ${ }^{5}$

Stark absorption measurements were performed on $\mathbf{1}^{-}$and $\mathbf{2}^{-}$ in the NIR region, at $77 \mathrm{~K}$, to determine the effective dipole moment change $\left(\Delta \mu_{12}\right)$ associated with intervalence excitation; $\Delta \mu_{12}$ can then be related to the adiabatic charge-transfer distance $\left(R_{12}\right)$ by

$$
\Delta \mu_{12}=R_{12} \times e
$$

where $e$ is the unit electron charge. Typically, for highly coupled MV systems, $R_{12}$ is significantly smaller than the geometric distance between redox centers

The 77 K NIR absorption and Stark spectra of $\mathbf{1}^{-}$and $\mathbf{2}^{-}$reveal a number of similarities in addition to several significant differences compared to the previously examined tetra-Re molecular species. The low temperature IT bands of $\mathbf{1}^{-}$and $\mathbf{2}^{-}$are slightly narrower and shifted to higher energy as compared to the room temperature spectra. Both of these effects are similar to previously reported molecular rectangles. ${ }^{3 \mathrm{~b}, \mathrm{c}}$ In contrast to the molecular rectangles though, the IT band absorption profiles deviate from simple Gaussian shapes, with the addition of a prominent shoulder, suggesting the presence of multiple overlapping transitions. The Stark spectra mirror this finding, displaying a double peaked profile centered around the band maximum. Qualitatively the Stark spectra resemble the second derivative component of the absorption spectra, signifying a measurable amount of charge transfer. Liptay

10.1021/ja0623764 CCC: $\$ 33.50$ @ 2006 American Chemical Society 


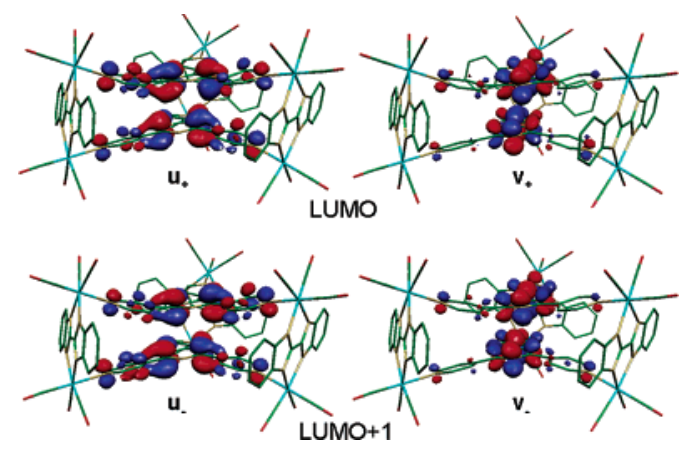

Figure 2. Side view of the frontier molecular orbitals determined at the DFT B3LYP/LanL2DZ level for $\mathbf{1}$.

analyses $^{6}$ of the Stark spectra, measured at three angles $\chi$, reveal small dipole moment changes $\left(\left|\Delta \mu_{12}\right|\right): 0.30 \pm 0.02 \mathrm{e} \AA$ for $\mathbf{1}^{-}$and $0.48 \pm 0.02 \mathrm{e}^{\mathrm{A}}$ for $\mathbf{2}^{-}$.

Estimation of the electronic coupling energy $\left(H_{\mathrm{ab}}\right)$ from eq 2 requires knowledge of $R_{\mathrm{ab}}$, the diabatic electron-transfer distance.

$$
H_{\mathrm{ab}}=\left(0.0206 / R_{\mathrm{ab}}\right) \sqrt{v_{\max } \times \Delta v_{1 / 2} \times \epsilon_{\max }}
$$

$R_{\mathrm{ab}}$ can be estimated from $R_{12}$ using the optical parameters of the IT bands by the generalized Mulliken-Hush analysis. ${ }^{7}$ This yields $R_{\mathrm{ab}}$ distances of 1.65 and $1.87 \AA$ and $H_{\mathrm{ab}}$ values of 2800 and 2600 $\mathrm{cm}^{-1}$ for $\mathbf{1}^{-}$and $\mathbf{2}^{-}$, respectively. Notably, the values for $R_{\mathrm{ab}}$ are significantly shorter than the geometrical distance between adjacent tPyTz ligands, and the values for $2 H_{\mathrm{ab}}$ are roughly equal to $v_{\max }$. Taken together, these data suggest that $\mathbf{1}^{-}$and $\mathbf{2}^{-}$are highly coupled MV systems that lie close to the Class II/III boundary. ${ }^{5}$

Returning to the Stark data, the most surprising difference between $\mathbf{1}^{-}$or $\mathbf{2}^{-}$and the previously reported molecular rectangles ${ }^{3}$ is the deviation from collinearity of the transition dipole moment and $\Delta \mu_{12}$ vectors (angle $\zeta$ ). The measured values of $\zeta$ for $\mathbf{1}^{-}$and $2^{-}$are $43 \pm 14$ and $45 \pm 10^{\circ}$, respectively. In other words, charge redistribution due to intervalence excitation is strongly skewed relative to the direction of light absorption (roughly the tPyTztPyTz axis), despite the apparent $D_{3 h}$ symmetry. This unexpected result is unique to the prisms. Of the MV compounds previously examined by Stark spectroscopy, including the tetra-Re rectangles, all exhibit collinear transition dipole and dipole moment change vectors (i.e., $\zeta=0^{\circ}$ ). This new result suggests that the donoracceptor states involved in the IT process of $\mathbf{1}^{-}$and $\mathbf{2}^{-}$are more complex than in conventional two-state systems.

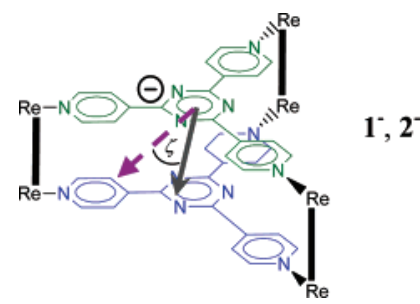

To explore this possibility and to understand better the nature of the intervalence excitation, density functional theory electronic structure calculations were performed on $\mathbf{1}$. These calculations confirm the through-space nature of electronic interactions between cofacial tPyTz ligands. The calculations carried out on the neutral structure reveal that in the case of trigonal symmetry the LUMO and LUMO+1 states (Figure 2) involved in the IT excitation are 2-fold degenerate (E state). The corresponding components (u and v) of these states represent symmetric (LUMO) and antisymmetric $(\mathrm{LUMO}+1)$ linear combinations of ligand-centered orbitals. Koop- man's theorem estimates for $H_{\mathrm{ab}}$, derived from calculations performed on pairs of tPyTz ligands (using the X-ray geometry) with and without the bis-Re edges, are $\sim 1460 \mathrm{~cm}^{-1}$ in both cases, illustrating that metal-mediated coupling is not significant. Similar results were obtained from semiempirical (ZINDO) calculations.

As a consequence of Jahn-Teller and low-symmetry crystalfield effects, the orbital degeneracy of the LUMO and LUMO+1 states is lifted. The four new electronic states can then be represented as linear combinations of the $\mathbf{u}$ and $\mathbf{v}$ components of the corresponding original E state. Note that, while the E state has no net dipole moment, any linear combination $a \mathbf{u}+b \mathbf{v}$ (with $a$ and $b$ being arbitrary constants), even in the case of trigonal symmetry, has a state dipole moment oriented in the plane of the tPyTz ligand. ZINDO calculations for a model system consisting of two tPyTz ligands indicate that, in contrast to conventional MV systems, the dipole moment of the adiabatic states is tilted away from the triazine-triazine axis. These results suggest that the unusual feature seen in the Stark spectra likely arises from the spatial properties of the orbital degrees of freedom.

Excited-state calculations carried out for a nearly trigonal symmetry of the model system show a single dipole-allowed chargertransfer band involving the $\mathrm{u}_{-}$and $\mathrm{u}_{+}$orbitals shown in Figure 2 . The calculations also indicate some build up of IT character and an increase in intensity for other transitions, as the dimer departs from a trigonal symmetry following a geometry distortion of the monomers or displacement of one of the partners.

However, further investigations of the adiabatic potential surfaces of $\mathbf{1}^{-}$and $\mathbf{2}^{-}$as well as excited-state calculations at a multiconfiguration level are needed for an unambiguous assignment of the observed NIR shoulder. Alternatively, the shoulder might have a vibrational origin, as is often seen for delocalized MV systems.

In conclusion, two triangular molecular prisms based on the tPyTz ligand have been characterized in the singly reduced state by NIR absorbance and Stark spectroscopy. The prisms, in their singly reduced form, comprise highly coupled MV species. The orbital degeneracy of the tPyTz redox sites has pronounced effects on light-induced (IT) charge redistribution within the molecules.

Acknowledgment. We gratefully acknowledge DOE's Office of Science (Grant DE-FG02-87ER13808) and the NSF (through the STC Program (DMR-0120967), the CRIF Program (CHE040443564), and Grant CHE-0342321) for financial support of work at NU and Georgia Tech, respectively.

Supporting Information Available: X-ray crystallographic file for 1, IT absorbance and electroabsorbance spectra, and experimental and computational details. This material is available free of charge via the Internet at http://pubs.acs.org.

\section{References}

(1) (a) Demadis, K. D.; Hartshorn, C. M.; Meyer, T. J. Chem. Rev. 2001 101, 2655-2685. (b) Crutchley, R. J. Adv. Inorg. Chem. 1994, 41, 273325 .

(2) See, for example: (a) Lambert, C.; Risko, C.; Coropceanu, V.; Schelter J.; Amthor, S.; Gruhn, N. E.; Durivage, J. C.; Bredas, J. L. J. Am. Chem. Soc. 2005, 127, 8508-8516. (b) Nelsen, S. F.; Konradsson, A. E.; Telo, J. P. J. Am. Chem. Soc. 2005, 127, 920-925. (c) Barlow, S.; Risko, C.; Chung, S. J.; Tucker, N. M.; Coropceanu, V.; Jones, S. C.; Levi, Z.; Bredas, J. L.; Marder, S. R. J. Am. Chem. Soc. 2005, 127, 16900-16911.

(3) (a) Dinolfo, P. H.; Williams, M. E.; Stern, C. L.; Hupp, J. T. J. Am. Chem. Soc. 2004, 126, 12989-13001. (b) Dinolfo, P. H.; Hupp, J. T. J. Am Chem. Soc. 2004, 126, 16814-16819. (c) Dinolfo, P. H.; Lee, S. J.; Coropceanu, V.; Brédas, J. L.; Hupp, J. T. Inorg. Chem. 2005, 44, 57895797.

(4) Hush, N. S. Prog. Inorg. Chem. 1967, 8, 391-444.

(5) Brunschwig, B. S.; Creutz, C.; Sutin, N. Chem. Soc. Rev. 2002, 31, 168184

(6) (a) Liptay, W. Angew. Chem., Int. Ed. Engl. 1969, 8, 177-188. (b) Liptay, W. In Excited States; Lim, E. C., Ed.; Academic Press: New York, 1974; Vol. 1, pp 129-229.

(7) Cave, R. J.; Newton, M. D. J. Chem. Phys. 1997, 106, 9213-9226. JA0623764 